\title{
Phase-locking between Kuramoto oscillators: robustness to time-varying natural frequencies
}

\author{
Alessio Franci, Antoine Chaillet and William Pasillas-Lépine
}

\begin{abstract}
In this paper we analyze the robustness to timevarying natural frequencies of phase-locking in the Kuramoto system with general interconnection topologies. This model allows to consider the effect of heterogeneity in the ensemble of oscillators, exogenous disturbances, and unmodeled dynamics on the behavior of the Kuramoto model. In particular, we prove the local input-to-state stability with respect to small inputs of all the asymptotically stable phase-locked states. The analysis, based on a Lyapunov function for the incremental dynamics of the system, provides a general methodology to build explicit bounds on the region of attraction, on the size of admissible inputs, and on the input-to-state gains. As an application of this method, we show that, in the particular case of the allto-all coupling, it allows to prove the exponential input-tostate stability of the synchronized state when all the initial phase differences lie in $\left[-\frac{\pi}{2}, \frac{\pi}{2}\right]$, and to provide an explicit bound on the convergence rate, thus extending recent results on the exponential synchronization of the finite Kuramoto model with constant different natural frequencies to the time-varying case. Furthermore, the proposed Lyapunov function for the incremental dynamics allows for a new characterization of the asymptotically stable phase-locked states of the unperturbed dynamics in terms of its isolated local minima.
\end{abstract}

\section{INTRODUCTION}

Synchronization has recently found many applications in the modeling and control of physical, chemical, medical, biological, and engineering problems cf. e.g. [1], [2], [3], [4], [5], [6], [7], [8]. Roughly speaking, an ensemble of interacting agents is said to synchronize when their outputs tend to a common value [22, Chapter 5]. Examples of such a behavior behavior can be found in interconnected neurons [5], [9], [10], chemical oscillators [8], coupled mechanical systems [11] and consensus algorithm [12], [13], [14].

In this paper we focus on phase-locking, or frequency locking, that is the synchronization between oscillating unities when their output is given by the frequency of oscillation. The use of mathematical models to understand phaselocking, started a few decades ago with the seminal works of Kuramoto [8] and Winfree [5]. Later on, many other works generalized their results [12], [15], [16], [17], [18], [19], [20], [21], [6], [14], [22], [23]. One of the most widely used model in the analysis of phase-locking between nonlinear coupled oscillators is the Kuramoto model [24], [16], [17], [18], [19], [20], [8]. In an other work [31] we address the problem of desynchronizing an ensemble of neurons through

\footnotetext{
Alessio Franci is with LSS-Université Paris Sud-Supélec, 3, rue Joliot-Curie, 91192 Gif-sur-Yvette, France, mail: Alessio.Franciels.supelec.fr

Antoine Chaillet is with EECI-LSS-Université Paris Sud-Supélec, mail: Antoine.Chailletesupelec.fr

William Pasillas-Lépine is with CNRS-LSS-Supélec, mail: William.Pasillas-Lepinedlss.supelec.fr
}

a scalar feedback (mean-field feedback). Motivated by the aim of computing necessary conditions on the feedback gain to obtain an effective desynchronization, in this paper we study the robustness of phase-locking in the Kuramoto model to time-varying inputs. In particular, we address the more general problem of robustness to time varying natural frequencies, which permits to encompass different types of perturbations: heterogeneities in the ensemble of oscillators, such as different natural frequencies, are known to prevent phase-locking, if the coupling strength is too small [18], [17], [32], [26], [23]; the system may be perturbed by exogenous inputs, such as noise [25], or deterministic signals, as in the case of the feedback approach to desynchronization [26], [27], [28], [29], [30], [31], which can affect in different ways, and even kill, phase-locking; there can be the presence of unmodeled dynamics, for example time-varying interconnection topologies, as in the case of neurons, where little is known about the real connectivity, which has to be considered (see [32] for a simulation study of this situation). For the sake of robustness, all these perturbations can be easily cast as time-varying natural frequencies (cf. e.g. (2)). This problem has been partially addressed in the infinite dimensional Kuramoto model (see [16] for a nice survey). This model permits an easier analytical treatment of the effect of external inputs, by allowing a linear partial integrodifferential equation for the order parameter of the system. This approach have been used to analyze the effect of delayed [30] and multisite [27] mean-field feedback approach to desynchronization. In the case of stochastic inputs it allows to find the critical coupling in the presence of noise [25]. On the other hand, the infinite dimensional model is well defined only in the case of all-to-all interconnection. In the finite case, [32] proposes a complete numerical analysis in the case of time-varying natural frequencies, time-varying interconnection topologies and non-sinusoidal coupling. Their results shows that phase-locking can show robustness to all this kind of perturbations. The same conclusion holds from the numerical simulations in [31] with respect to mean-field feedback. Analytical studies on the robustness of phase-locking in the finite Kuramoto model have been addressed only for constant inputs. Necessary and sufficient conditions for the existence of a stable phase-locked state in the Kuramoto model with general interconnection topologies and constant different natural frequencies can be found in [18]. Necessary and sufficient conditions for the exponential synchronization of the Kuramoto system with the all-toall coupling and constant different natural frequencies can be found in [15]. The existence and explicit expression of 
the fixed points describing stable and unstable phase-locked states in the case of all-to-all coupling with constant different natural frequencies and as a function of the coupling strength is studied in [17]. By the way, we cite [19], where for the first time a Lyapunov function for the perturbed Kuramoto system with the all-to-all coupling was proposed in order to analyze the local stability of phase-locking. To the best of our knowledge the problem of the robustness of phaselocking with respect to time-varying natural frequencies has still not been analytically addressed in the finite Kuramoto model.

The aim of this paper is then to study the robustness to time-varying natural frequencies of phase-locked states in the finite Kuramoto model with general interconnection topologies

$$
\dot{\theta}_{i}(t)=\varpi_{i}(t)+\sum_{j=1}^{N} k_{i j} \sin \left(\theta_{j}(t)-\theta_{i}(t)\right), \forall t \geq 0,
$$

for all $i=1, \ldots, N$, where $\theta_{i} \in \mathrm{T}^{1}$ denotes the phase of each oscillator, $\varpi_{i}: \mathbb{R}_{\geq 0} \rightarrow \mathbb{R}$ denotes its time-varying natural natural frequency, and $k=\left[k_{i j}\right]_{i, j=1, \ldots, N} \in \mathbb{R}_{\geq 0}^{N \times N}$ is the coupling matrix ${ }^{1}$. This model allows to consider the influence of the heterogeneity of the ensemble, of external disturbances (such as the mean-field feedback introduced in [31]), and of unmodeled dynamics (time-varying interconnection topology, non-sinusoidal coupling, etc.), on the behavior of the Kuramoto model. For example, (1) encompasses uncertainties and time evolution of the interconnection topology, which we denote by $\delta k_{i j}(t)$, for all $i, j=1, \ldots, N$ and all $t \geq 0$, by letting

$$
\varpi_{i}(t)=\sum_{j=1}^{N} \delta k_{i j}(t) \sin \left(\theta_{j}(t)-\theta_{i}(t)\right), \forall i=1, \ldots, N, t \geq 0 .
$$

A similar construction holds for non-sinusoidal coupling functions and exogenous inputs.

It results that phase-locking in the Kuramoto model is locally input-to-state stable (ISS) with respect to small inputs. This result is not restricted to the perfectly synchronized state, but to any other asymptotically stable phase-locked state which may appear for interconnection topologies different from the all-to-all coupling. These states are characterized by all the unities oscillating at the same frequency, but with nonzero phase differences. Examples of this behavior have been recently shown in [14, Chapter 3]. The proof of the main result is based on the existence of an ISS-Lyapunov function for the incremental dynamics of the system. This analysis provides a general methodology to build explicit estimates on the size of the region of convergence, the ISS gain and the tolerated input bound, which applies to general symmetric interconnection topologies and to any asymptotically stable phase-locked state. As an application of the main result, we restrict our analysis to the all-to-all interconnection topology. Recently, the exponential synchronization of the Kuramoto

\footnotetext{
${ }^{1}$ See the Notation section below for the meaning of the used symbols.
}

system with constant different natural frequencies and all-toall coupling have been shown in [15]. Our analysis permits to extend this result to the time-varying case. In particular, we are able to prove the exponential input-to-state stability of synchronization when all the initial phase differences lie in $\left[-\frac{\pi}{2}, \frac{\pi}{2}\right]$, and give explicit bounds on the convergence rate. To show the reliability of our method, the size of the region of convergence, the sufficient bound on the coupling strength and the convergence rate are compared to those obtained in [15]. Furthermore, the Lyapunov function for the incremental dynamics allows for a new characterization of the asymptotically stable phase-locked states of the unperturbed dynamics. Indeed, the invariance of the Kuramoto dynamics with respect to common phase shift among the ensemble (i.e. $S^{1}$ symmetry), implies the existence of an invariant manifold for the unperturbed system. We show that asymptotically stable phase-locked states correspond to isolated local minima of the proposed Lyapunov function restricted to this manifold.

\section{Notations}

For a set $A \subset \mathbb{R}$, and $a \in A, A_{\geq a}$ denotes the set $\{x \in$ $A: x \geq a\} .|x|$ denotes the Euclidean norm of the vector $x$, that is, if $x \in \mathbb{R}^{n},|x|:=\sqrt{\sum_{i=1}^{n} x_{i}^{2}}$. For a subset $\mathcal{A} \subset \mathbb{R}^{n}$, and $x \in \mathbb{R}^{n},|x|_{\mathcal{A}}=\inf _{y \in \mathcal{A}}|y-x|$ denotes the point-toset distance from $x \in \mathbb{R}^{n}$ to $\mathcal{A} \subset \mathbb{R}^{n}$. $\mathcal{B}(x, R)$ refers to the closed ball of radius $R$ centered at $x$ in the Euclidean norm, i.e. $\mathcal{B}(x, R):=\left\{z \in \mathbb{R}^{n}:|x-z| \leq R\right\}$; for a subset $\mathcal{A} \subset \mathbb{R}^{n}, \mathcal{B}(\mathcal{A}, R):=\left\{z \in \mathbb{R}^{n}:|z|_{\mathcal{A}} \leq R\right\}$. $\|u\|$ is the $L^{1}$ norm of the signal $u(\cdot)$, that is, if $u: \mathbb{R}_{\geq 0} \rightarrow$ $\mathbb{R}^{n}$ denotes a measurable signal, locally essentially bounded, $\|u\|:=\operatorname{esssup}_{t>0}|u(t)|$. A continuous function $\alpha: \mathbb{R}_{\geq 0} \rightarrow$ $\mathbb{R}_{\geq 0}$ is said to be of class $\mathcal{K}$ if it is increasing and $\alpha(0)=0$. It is said to be of class $\mathcal{K}_{\infty}$ if it is of class $\mathcal{K}$ and $\alpha(s) \rightarrow \infty$ as $s \rightarrow \infty$. A function $\beta: \mathbb{R}_{\geq 0} \times \mathbb{R}_{\geq 0} \rightarrow \mathbb{R}_{\geq 0}$ is said to be of class $\mathcal{K} \mathcal{L}$ if $\beta(\cdot, t) \in \mathcal{K}$ for any fixed $t \geq 0$ and $\beta(s, \cdot)$ is continuous decreasing and tends to zero at infinity for any fixed $s \geq 0$. $\mu^{n}$ denotes the Lebesgue measure on $\mathbb{R}^{n} . \mathrm{T}^{n}$ denotes the n-Torus. If $x \in \mathbb{R}^{n}, \nabla_{x}$ is the gradient vector with respect to $x$, i.e. $\nabla_{x}=\left(\frac{\partial}{\partial x_{1}}, \ldots, \frac{\partial}{\partial x_{n}}\right)$. Given $x \in \mathbb{R}^{n}$ and $a \in \mathbb{R},(x \bmod a)=\left[x_{i} \bmod a\right]_{i=1, \ldots, n}$, where mod denotes the modulo operator. The vector with all unitary components in $\mathbb{R}^{n}$ is denoted by $\mathbf{1}_{n}$.

\section{ROBUSTNESS OF PHASE-LOCKED SOLUTIONS}

\section{A. Robustness analysis}

The robustness analysis of phase-locked states can be translated in the study of the fixed points of the incremental dynamics $\dot{\theta}_{i}-\dot{\theta}_{j}$ associated to (1). The same approach has been exploited in [15] in the case of all-to-all coupling and constant inputs. In contrast to [18], studying the incremental dynamics of the system avoids the use of the grounded Kuramoto model, in which the mean frequency of the ensemble is "grounded" to zero and synchronization corresponds to a fixed point. While the latter is a well defined mathematical object for constant perturbations, its extension to time-varying inputs, which is the subject of the present 
study, is not clear. The intuitive idea is that the incremental dynamics remains unchanged if the same input is added to each oscillator. Hence, we start by defining the common drift $\bar{\omega}$ of the system (1) as

$$
\bar{\omega}(t)=\frac{1}{N} \sum_{j=1}^{N} \varpi_{j}(t), \quad \forall t \geq 0
$$

and the grounded input $\tilde{\omega}$ as $\tilde{\omega}:=\left[\tilde{\omega}_{i}\right]_{i=1, \ldots, N}$, where

$$
\tilde{\omega}_{i}(t):=\varpi_{i}(t)-\bar{\omega}(t), \quad \forall i=1, \ldots, N, \forall t \geq 0 .
$$

Noticing that $\varpi_{i}-\varpi_{j}=\tilde{\omega}_{i}-\tilde{\omega}_{j}$, the evolution equation of the incremental dynamics ruled by (1) reads

$$
\begin{gathered}
\dot{\theta}_{i}(t)-\dot{\theta}_{j}(t)=\tilde{\omega}_{i}(t)-\tilde{\omega}_{j}(t)+ \\
\sum_{l=1}^{N} k_{i l} \sin \left(\theta_{l}(t)-\theta_{i}(t)\right)-\sum_{l=1}^{N} k_{j l} \sin \left(\theta_{l}(t)-\theta_{j}(t)\right)
\end{gathered}
$$

for all $i, j=1, \ldots, N, i \neq j$, and all $t \geq 0$. As expected, the incremental dynamics is invariant with respect to common drifts among the ensemble of oscillators ${ }^{2}$. In the sequel we use $\tilde{\theta}$ to denote the incremental variable

$$
\tilde{\theta}:=\left[\theta_{i}-\theta_{j}\right]_{i, j=1, \ldots, N, i \neq j} \in \mathrm{T}^{(N-1)^{2}} .
$$

When no inputs are applied, i.e. $\tilde{\omega}=0$, we expect the solutions of (5) to converge to some asymptotically stable fixed point or, equivalently, the solution of (1) to converge to some asymptotically stable phase-locked solution. For that reason, we start by defining the notion of 0-asymptically stable (0-AS) phase-locked solutions, which, roughly speaking, is described by an asymptotically stable fixed point of the incremental dynamics (5) when no inputs are applied.

Definition 1 (0-AS phase-locked solutions) Given any coupling matrix $k \in \mathbb{R}_{\geq 0}^{N \times N}$, let $\mathcal{O}_{k}$ denote the set of asymptotically stable fixed points of the unperturbed (i.e. $\tilde{\omega} \equiv 0$ ) incremental dynamics (5). A phase-locked solution $\theta^{*}$ of (1) is said to be 0-asymptotically stable if and only if the incremental state $\tilde{\theta}^{*}:=\left[\left(\theta^{*}\right)_{i}-\left(\theta^{*}\right)_{j}\right]_{i, j=1, \ldots, N, i \neq j}$ belongs to $\mathcal{O}_{k}$.

A complete characterization of 0-AS phase-locked solutions of (1) for general interconnection topologies can be found in [33] and [14, Chapter 3]. In Section II-D, we characterize the set $\mathcal{O}_{k}$ in terms of the isolated local minima of a suitable Lyapunov function (cf. Remark 2 below).

The reason for considering only asymptotically stable fixed points of the incremental dynamics lies on the fact that only those are expected to provide some robustness properties (as asymptotic stability implies local robustness with respect to small inputs [34], [37]). On the contrary, stable fixed points may correspond to non-robust phase-locked state, as illustrated by the following example.

\footnotetext{
${ }^{2}$ This fact can also be interpreted as the invariance of the system (1) with respect to common phase-shift of the ensemble (i.e. $\theta_{i} \rightarrow \theta_{i}+c, \forall i=$ $1, \ldots, N)$. See for example [12].
}

Example 1 (Non robust phase-locking) Consider the case where $N>2$ and let $k_{12}=k_{21}>0$, and $k_{i j}=0$ for all $(i, j) \in \mathbb{N}_{\leq N} \times \mathbb{N}_{\leq N} \backslash\{(1,2),(2,1)\}$. When $\tilde{\omega}=0$, (5) then becomes

$$
\begin{gathered}
\dot{\theta}_{i}-\dot{\theta}_{j}=0 \\
\text { for all }(i, j) \in \mathbb{N}_{\leq N} \times \mathbb{N}_{\leq N} \backslash\{(1,2),(2,1)\} \text {, and } \\
\dot{\theta}_{1}-\dot{\theta}_{2}=-2 k_{12} \sin \left(\theta_{1}-\theta_{2}\right)
\end{gathered}
$$

In this case, all the solutions of the form $\theta_{1}(t)-\theta_{2}(t)=$ 0 , for all $t \geq 0$, and $\theta_{i}(t)-\theta_{j}(t)=\theta_{i}(0)-\theta_{j}(0)$, for all $t \geq 0$ and all $(i, j) \in \mathbb{N}_{\leq N} \times \mathbb{N}_{\leq N} \backslash\{(1,2),(2,1)\}$, are stable, but not asymptotically. By adding any (arbitrarily small) constant inputs $\tilde{\omega}_{l} \neq 0$, for some $l \in \mathbb{N}_{\leq N} \backslash\{1,2\}$, the system becomes completely desynchronized, since $\dot{\theta}_{l}-\dot{\theta}_{i} \equiv$ $\tilde{\omega}_{l}$ for all $i=1, \ldots, N, i \neq l$. In particular, in this case the set $\mathcal{O}_{k}$ is empty.

We next recall the definition of local Input-to-State Stability with respect to small inputs [35]. This concept is also referred to as Total Stability [37].

Definition 2 (LISS w.r.t. small inputs) For a system of the form $\dot{x}=f(x, u)$, a set $\mathcal{A} \subset \mathbb{R}^{n}$ is if there exist $\delta, \delta_{u}>0$, a class $\mathcal{K} \mathcal{L}$ function $\beta$ and a class $\mathcal{K}_{\infty}$ function $\rho$, such that, for all $\left|x_{0}\right|_{\mathcal{A}} \leq \delta_{x}$ and all $u$ satisfying $\|u\| \leq \delta_{u}$, its solution satisfies

$$
|x(t)|_{\mathcal{A}} \leq \beta\left(\left|x_{0}\right|_{\mathcal{A}}, t\right)+\rho(\|u\|), \quad \forall t \geq 0 .
$$

If the same estimate holds with $\beta(r, s)=C r e^{-\frac{s}{\tau}}$, where $C, \tau$ are positive constants, then the $\mathcal{A}$ is said to be locally exponentially Input-to-State Stable with respect to small inputs,

Remark 1 (Local Euclidean metric on the n-Torus)

Definition 2 is given on $\mathbb{R}^{n}$, which is little adapted to the context of this article. Its extension to the n-Torus is natural since $\mathrm{T}^{n}$ is locally isometric to $\mathbb{R}^{n}$ through the identity map $\mathcal{I}$ (i.e. $|\theta|_{\mathrm{T}^{n}}:=|\mathcal{I}(\theta)|=|\theta|$ ). In particular this means that we are providing the n-Torus with the local Euclidean metric and its induced norm. Hence, Definition 2 applies locally in the n-Torus.

The next theorem, whose proof is given in Section III-A, states the LISS of $\mathcal{O}_{k}$ with respect to small inputs.

Theorem 1 (LISS w.r.t. small inputs of phase-locking) Let $k$ be any symmetric interconnection matrix in $\mathbb{R}_{\geq 0}^{N \times N}$. Suppose that the set $\mathcal{O}_{k}$ is non-empty. Then there exist $\delta_{\tilde{\theta}}, \delta_{\omega}>0$, a class $\mathcal{K} \mathcal{L}$ function $\beta$ and a class $\mathcal{K}_{\infty}$ function $\rho$, such that the solution of (5) satisfies, for all $\tilde{\omega}$ satisfying $\|\tilde{\omega}\| \leq \delta_{\omega}$ and all $\tilde{\theta}_{0} \in \mathcal{B}\left(\mathcal{O}_{k}, \delta_{\tilde{\theta}}\right)$,

$$
|\tilde{\theta}(t)|_{\mathcal{O}_{k}} \leq \beta\left(\left|\tilde{\theta}_{0}\right|_{\mathcal{O}_{k}}, t\right)+\rho(\|\tilde{\omega}\|), \forall t \geq 0 .
$$

Theorem 1 guarantees that, if a configuration is asymptotically stable for the unperturbed system, then solutions starting sufficiently near from that configuration remain near it at all time, providing that the perturbation $\tilde{\omega}$ is sufficiently 
small. Moreover the distance of the incremental state from $\mathcal{O}_{k}$ is somehow "proportional" to the amplitude of $\tilde{\omega}$ with nonlinear gain $\rho$. This means that the phase-locked states described by $\mathcal{O}_{k}$ are robust to external inputs, provided they are not too large. We stress that, while local ISS with respect to small inputs is a natural consequence of asymptotic stability [34], the size of the constants $\delta_{x}$ and $\delta_{u}$ in Definition 2, defining the robustness zone in terms of initial conditions and inputs amplitude, are potentially infinitesimal. As we show explicitly in the next section in the special case of all-to-all coupling, the Lyapunov analysis used in the proof of Theorem 1 (cf. Section III-A) provides a general methodology to build these estimates explicitly. We stress in particular that, while the region of attraction is fixed by the geometric properties of the fixed points of the unperturbed system (cf. (23) and (25)), the size of admissible inputs can be made large at will by taking a sufficiently large coupling strength (cf. (28) and (29)).

B. Robustness of the synchronized state in the case of allto-all coupling

In this section we focus the Lyapunov analysis used in the proof of Theorem 1 to the case of all-to-all coupling. In this case, the only asymptotically stable phase-locked state is the synchronization, that is the state characterized by zero phase difference between each couple of oscillators [33]. The following proposition states the exponential input-tostate stability of the synchronized state with respect to small inputs. In particular it gives explicit bounds on the region of convergence, the size of admissible inputs, the ISS gain, and the convergence rate. Its proof can be found in Section III-B.

Proposition 1 (Exponential LISS of synchronization) Consider the system (1) with the all-to-all interconnection topology, i.e. $k_{i j}=K>0$ for all $i, j=1, \ldots, N$. Then, for all $0 \leq \epsilon \leq \frac{\pi}{2}$, and all $\tilde{\omega}$ satisfying

$$
\|\tilde{\omega}\| \leq \delta_{\omega}^{\epsilon}:=\frac{K \sqrt{N}}{\pi^{2}}\left(\frac{\pi}{2}-\epsilon\right),
$$

the following facts hold:

1) The set $\mathcal{D}_{\epsilon}:=\left\{\tilde{\theta}: \max _{i, j=1, \ldots, N}\left|\theta_{i}-\theta_{j}\right| \leq \frac{\pi}{2}-\epsilon\right\}$ is forward invariant for the system (5).

2) For all $\tilde{\theta}_{0} \in \mathcal{D}_{0}$, the solution of (5) satisfies

$$
|\tilde{\theta}(t)| \leq \frac{\pi}{2}\left|\tilde{\theta}_{0}\right| e^{-\frac{K}{\pi^{2}} t}+\frac{\pi^{2}}{K}\|\tilde{\omega}\|
$$

and the set $\mathcal{D}_{\epsilon}$ is attractive over $\mathcal{D}_{0}$.

Proposition 1 establishes the exponential ISS of the synchronized state in the Kuramoto model with the all-to-all interconnection topology with respect to time-varying inputs whose amplitude is smaller than $\frac{K \sqrt{N}}{2 \pi}$ and for any initial condition lying in $\mathcal{D}_{0}$, that is when all the initial phase differences lie in $\left[-\frac{\pi}{2}, \frac{\pi}{2}\right]$. Moreover, if the inputs amplitude is bounded by $\delta_{\omega}^{\epsilon}$, for some $0 \leq \epsilon \leq \frac{\pi}{2}$, then the set $\mathcal{D}_{\epsilon}$ is forward invariant and attractive for all initial conditions in $\mathcal{D}_{0}$. Recently, necessary and sufficient conditions for the exponential synchronization of the Kuramoto system with all-to-all coupling and constant different natural frequencies were given in [15]. We stress that the estimated region of attraction provided by Proposition 1 is strictly larger than the one obtained in [15, Theorem 4.1], which does not allow $\epsilon$ to be picked as zero. For initial conditions lying in $\mathcal{D}_{\epsilon}$, with a strictly positive $\epsilon$, we can compare the convergence rate obtained in Proposition $1, \frac{K}{\pi^{2}}$, with the one obtained in [15, Theorem 3.1], $N K \sin (\epsilon)$. While the convergence rate of Proposition 1 is slower than the one obtained in [15, Theorem 3.1] for large $\epsilon$ (i.e. small region of attraction), it provides a better estimate for small values of $\epsilon$ (that is for a large domain of attraction). The inputs amplitude bound (7) allows to find the sufficient coupling strength $K_{\epsilon}$ which let the set $\mathcal{D}_{\epsilon}$ be invariant, and attractive, when $\|\tilde{\omega}\| \leq \delta_{\omega}$, for some $\delta_{\omega}>0$,

$$
K_{\epsilon}=\frac{\pi^{2}}{\left(\frac{\pi}{2}-\epsilon\right) \sqrt{N}}\|\tilde{\omega}\| .
$$

Noticing that $\sqrt{N} \max _{i, j=1, \ldots, N}\left\|\varpi_{i}-\varpi_{j}\right\| \geq\|\tilde{\omega}\|$, we get that

$$
K_{\epsilon} \leq \frac{\pi^{2}}{\left(\frac{\pi}{2}-\epsilon\right)} \max _{i, j=1, \ldots, N}\left\|\varpi_{i}-\varpi_{j}\right\| .
$$

Since $\left(\frac{\pi}{2}-\epsilon\right) \geq \frac{2}{\pi} \cos (\epsilon)$, for all $0 \leq \epsilon \leq \frac{\pi}{2}$, it results that

$$
K_{\epsilon} \leq \frac{\pi^{3}}{2 \cos (\epsilon)} \max _{i, j=1, \ldots, N}\left\|\varpi_{i}-\varpi_{j}\right\|<\pi^{3} K_{i n v}
$$

where $K_{\text {inv }}$ is the sufficient coupling strength to let $\mathcal{D}_{\epsilon}$ be invariant as computed in [15, Proof of Theorem 4.1]. This observation shows that $K_{\epsilon}$ is of the same order of $K_{i n v}$, in the sense that $\frac{K_{\epsilon}}{K_{i n v}}<\pi^{3}$.

In conclusion, Proposition 1 partially extends the main results of [15] to time varying inputs. On the one hand, it allows to consider a larger set of initial conditions, and bounds the convergence rate to a strictly positive value, independently of the region of attraction. On the other hand it may require larger coupling strength, and, for small region of attractions, the bound on the convergence rate obtained in Proposition 1 is not as good as the one of [15, Theorem 3.1].

C. Robustness of neural synchrony to mean-field feedback Deep Brain Stimulation

The model (1) encompasses our model of interconnected neurons under mean-field feedback DBS, introduced in [31] and that is referred to as the Kuramoto system under real mean-field feedback,

$$
\begin{gathered}
\dot{\theta}_{i}(t)=\omega_{i}+\sum_{j=1}^{N} k_{i j} \sin \left(\theta_{j}-\theta_{i}\right)+ \\
\sum_{j=1}^{N} \gamma_{i j} \sin \left(\theta_{j}(t)-\theta_{i}(t)\right)-\sum_{j=1}^{N} \gamma_{i j} \sin \left(\theta_{j}(t)+\theta_{i}(t)\right)
\end{gathered}
$$

for all $i=1, \ldots, N$ and all $t \geq 0$, where $\omega_{i}$ is the constant natural frequency of the $i$-th neuron, $k=\left[k_{i j}\right]_{i, j=11, \ldots, N} \in$ $\mathbb{R}^{N \times N}$ describes the interconnection between the neurons in 
the subthalamic nucleus, and $\gamma=\left[\gamma_{i j}\right]_{i, j=11, \ldots, N} \in \mathbb{R}^{N \times N}$ is the feedback gain. Indeed, the effect of the real mean-field feedback can be modeled as an exogenous input, that is with

$$
\begin{gathered}
\varpi_{i}(t)= \\
\omega_{i}+\sum_{j=1}^{N} \gamma_{i j} \sin \left(\theta_{j}(t)-\theta_{i}(t)\right)-\sum_{j=1}^{N} \gamma_{i j} \sin \left(\theta_{j}(t)+\theta_{i}(t)\right),
\end{gathered}
$$

for all $i=1, \ldots, N$ and all $t \geq 0$. In particular the results of this paper can be used to compute necessary conditions on the mean field feedback gain to guarantee an effective desynchronization. To that end, consider the system (8), and define

$$
\bar{\gamma}:=\max _{i, j=1, \ldots, N}\left|\gamma_{i j}\right|
$$

i.e. $\bar{\gamma}$ denotes the intensity of the mean-field feedback DBS, and

$$
\omega^{\perp}:=\left[\omega_{i}-\frac{1}{N} \sum_{j=1}^{N} \omega_{j}\right]_{i=1, \ldots, N},
$$

i.e. $\omega^{\perp}$ represents the heterogeneity of the ensemble of neurons. We define the grounded mean-field input $\tilde{I}_{M F}$ of the incremental dynamics associated to (8) as

$$
\tilde{I}_{M F}(t):=I_{M F}(t)-\bar{I}_{M F}(t), \quad \forall t \geq 0
$$

where

$$
I_{M F}(t):=
$$

$\left[\sum_{j=1}^{N} \gamma_{i j}\left(\sin \left(\theta_{i}(t)-\theta_{j}(t)\right)-\sin \left(\theta_{i}(t)+\theta_{j}(t)\right)\right]_{i=1, \ldots, N}\right.$,

for all $t \geq 0$, represents the input of the mean-field feedback (cf. (9)) and

$\bar{I}_{M F}(t):=\frac{1}{N} \sum_{i, j=1}^{N} \gamma_{i j}\left(\sin \left(\theta_{i}(t)-\theta_{j}(t)\right)-\sin \left(\theta_{i}(t)+\theta_{j}(t)\right)\right.$, for all $t \geq 0$, represents the common drift among the ensemble of neurons due to the mean-field feedback. The grounded mean-field input $\tilde{I}_{M F}$ is the quantity of interest for the aim of desynchronization. Indeed, as already pointed out in (5), it is this input, along with the intrinsic heterogeneity of the ensemble (i.e. $\omega^{\perp}$ ), which is responsible for the destabilization of the incremental dynamics of the Kuramoto system under real mean-field feedback. The following result underlines the robustness of phase-locking to this particular perturbation. In other words, it provides a negative answer to the question whether mean-field feedback stimulation with arbitrarily small amplitude can effectively desynchronize the STN neurons.

Corollary 1 Let $k \in \mathbb{R}_{\geq 0}^{N \times N}$ be any symmetric interconnection matrix and $\left\{\omega_{i}\right\}_{i=1, \ldots, N}$ be any (constant) natural frequencies. Let $\gamma \in \mathbb{R}^{N \times N}$ be any feedback gain. Let $\bar{\gamma}$ and $\omega^{\perp}$ be defined as in (10)-(11). Let the set $\mathcal{O}_{k}$ be defined as in Definition 1 and suppose that it is non-empty. Then there exist a class $\mathcal{K} \mathcal{L}$ function $\beta$, a class $\mathcal{K}_{\infty}$ function $\sigma$, a positive constant $\delta_{\omega}$, and a neighborhood $\mathcal{P}$ of $\mathcal{O}_{k}$, such that, for all natural frequencies and all mean-field feedback satisfying

$$
\left|\omega^{\perp}\right|+2 \bar{\gamma} N \sqrt{N} \leq \delta_{\omega},
$$

the solution of the incremental dynamics of (8) satisfies, for all $\tilde{\theta}_{0} \in \mathcal{P}$,

$$
|\tilde{\theta}(t)|_{\mathcal{O}_{k}} \leq \beta\left(\left|\tilde{\theta}_{0}\right|_{\mathcal{O}_{k}}, t\right)+\sigma\left(\left|\omega^{\perp}\right|+\left\|\tilde{I}_{M F}\right\|\right),
$$

where $\tilde{I}_{M F}$ is defined in (12).

Corollary 1 states that the phase-locked states associated to any symmetric interconnection topology are robust to sufficiently small real mean-field feedbacks. The intensity of the tolerable feedback gain $\bar{\gamma}$ depends on the distribution of natural frequencies, reflecting the fact that a heterogeneous ensemble can be more easily brought to an incoherent state.

Energy consumption is a critical issue in the DBS framework [38]. Corollary 1, along with the explicit input bound $\delta_{\omega}$, which can be found in the proof of Theorem 1 (cf. (29)), provide a necessary condition on the intensity of the DBS through mean-field feedback to achieve effective desynchronisation for a general interconnection between neurons and recording-stimulation setup. Even an approximate knowledge of the distribution of natural frequencies of the neurons in the STN, of their interconnection topology and of the electrical characteristics of the recording-stimulation setup can be used to compute this value, based on the Lyapunov analysis detailed in Section II-D.

Future works will focus on the computation of sufficient conditions on this intensity in order to achieve full desynchronization (see [27] for the $N \rightarrow \infty$ case with all-to-all coupling and separate stimulation-registration setup).

\section{A Lyapunov function for the incremental dynamics}

In this section, we introduce the Lyapunov function for the incremental dynamics (5) used in the proof of Theorem 1. Beyond the technical interest of the use of this function, it provides a new characterization of the asymptotically stable phase-locked solutions and helps to partially extend existing results on the robustness of phase-locking in the finite Kuramoto model. Let

$$
E=\left[E_{i j}\right]_{i, j=1, \ldots, N}:=\frac{1}{K}\left[k_{i j}\right]_{i, j=1, \ldots, N},
$$

where $K=\max _{i, j=1, \ldots, N} k_{i j}$, denote the normalized interconnection matrix associated to $k$. Inspired by [14, Chapter 3], we consider the incremental Lyapunov function $V_{I}$ : $\mathrm{T}^{(N-1)^{2}} \rightarrow \mathbb{R}_{\geq 0}$ defined by

$$
V_{I}(\tilde{\theta})=2 \sum_{i=1}^{N} \sum_{j=1}^{N} E_{i j}\left(\sin \left(\frac{\theta_{i}-\theta_{j}}{2}\right)\right)^{2}
$$

where the incremental variable $\tilde{\theta}$ is defined in (6). We stress that $V_{I}$ is independent of the coupling strength $K$.

Before detailing the behavior of this function along the solution of (5), we underline the following issue. The components of the incremental variable $\tilde{\theta}$ are not linearly independent. Indeed, we can express $(N-1)(N-2)$ of them in terms of the other $N-1$ independent components. By choosing $\varphi_{i}:=\theta_{i}-\theta_{N}, i=1, \ldots, N-1$ as the independent variables, it is possible to write

$$
\begin{array}{rlrl}
\theta_{i}-\theta_{N} & =\varphi_{i}, & \forall i & =1, \ldots, N, \\
\theta_{i}-\theta_{j} & =\varphi_{i}-\varphi_{j}, \forall i & =1, \ldots, N, \forall j=1, \ldots, N-1 .(15 \mathrm{~b})
\end{array}
$$


These relations can be expressed in a compact form as

$$
\tilde{\theta}=\tilde{B}(\varphi):=B \varphi \quad \bmod 2 \pi, \quad \varphi \in \mathcal{M}
$$

where $\varphi:=\left[\varphi_{i}\right]_{i=1, \ldots, N-1}, B \in \mathbb{R}^{(N-1)^{2} \times(N-1)}, \operatorname{rank} B=$ $N-1, \tilde{B}$ is continuous and continuously differentiable, and $\mathcal{M} \subset \mathrm{T}^{(N-1)^{2}}$ is a submanifold defined by the embedding (16). The continuous differentiability of $\tilde{B}: \mathcal{M} \rightarrow$ $\mathrm{T}^{(N-1)^{2}}$ comes from the fact that $\varphi_{i} \in \mathrm{T}^{1}$, for all $i=$ $1, \ldots, N$, and the components of $\tilde{B}(\varphi)$ are linear functions of the form (15). Formally, this means that the system is evolving in the submanifold $\mathcal{M} \subset \mathrm{T}^{(N-1)^{2}}$ of dimension $(N-1)$. In particular $\mathcal{M}$ is diffeomorphic to $\mathrm{T}^{N-1}$.

In order to conduct Lyapunov analysis based on $V_{I}$ it is important to identify its critical points. By the fact that the system is evolving on the invariant manifold $\mathcal{M}$, only the critical points of the Lyapuonov function $V_{I}$ restricted to this manifold are of interest. That is, we have to restrict our attention to the critical points of the restriction of $V_{I}$ to $\mathcal{M}$, which is defined by the function $\left.V_{I}\right|_{\mathcal{M}}: \mathrm{T}^{N-1} \rightarrow \mathbb{R}$ as

$$
\left.V_{I}\right|_{\mathcal{M}}(\varphi):=V_{I}(B \varphi), \quad \forall \varphi \in \mathcal{M}
$$

Note that we can express the incremental variable $\varphi$ in terms of $\theta$ by means of a linear transformation $A \in \mathbb{R}^{(N-1) \times N}$, with $\operatorname{rank} A=N-1$, in such a way that

$$
\varphi=\tilde{A}(\theta)=A \theta \bmod 2 \pi \text {. }
$$

To make a clear link between the incremental variables and the actual phase of the neurons, define the function $V: \mathrm{T}^{N} \rightarrow \mathbb{R}$ as

$$
V(\theta)=\left.V_{I}\right|_{\mathcal{M}}(A \theta) .
$$

In contrast with $\left.V_{I}\right|_{\mathcal{M}}$, the function $V$ owns the advantage that its critical points are already widely studied in the synchronization literature, see for instance [6, Section III] and [14, Chapter 3]. The following lemma allows to reduce the analysis of the critical points of $V_{I}$ on $\mathcal{M}$ to that of the critical points of $V$ on $T^{N}$. Its proof is given in Section III-D.

Lemma 1 (Critical points of the incremental Lyapunov function) Let $\mathcal{M},\left.V_{I}\right|_{\mathcal{M}}, A$ and $V$ be defined as in (16)(19). Then $\theta^{*} \in T^{N}$ is a critical point of $V$ (i.e $\nabla_{\theta} V\left(\theta^{*}\right)=$ $0)$ if and only if $\varphi^{*}=A \theta^{*} \in \mathcal{M}$ is a critical point of $\left.V_{I}\right|_{\mathcal{M}}$ (i.e. $\left.\nabla_{\varphi} V_{I}\right|_{\mathcal{M}}\left(\varphi^{*}\right)=0$ ). Moreover if $\theta^{*}$ is a local maximum (resp. minimum) of $V$ then $\varphi^{*}$ is a local maximum (resp. minimum) of $\left.V_{I}\right|_{\mathcal{M}}$. Finally the origin of $\mathcal{M}$ is a local minimum of $\left.V_{I}\right|_{\mathcal{M}}$.

Another advantage of studying the incremental dynamics is that the critical points of $\left.V_{I}\right|_{\mathcal{M}}$ correspond to a unique phase-locked configuration, as opposed to the critical points of $V$ which correspond to phase-locked states modulo a common phase shift. The extra dimension of the non-incremental dynamics corresponds to the eigenvector $\mathbf{1}_{N}$ of the only zero eigenvalue of the non-incremental dynamics [17], [6]. The span of this vector describes common phase shift among the ensemble of oscillators.

The following lemma creates a bridge between the critical points of the incremental Lyapunov function and the fixed points of the incremental dynamics. In particular it allows to completely characterize the fixed points of the unperturbed incremental dynamics in terms of the critical points of $\left.V_{I}\right|_{\mathcal{M}}$ through the linear relations (15).

Lemma 2 (Incremental Lyapunov characterization of phase-locking) Let $k \in \mathbb{R}_{\geq 0}^{N \times N}$ be any symmetric interconnection matrix. Let $B$ and $\left.V_{I}\right|_{\mathcal{M}}$ be defined as in (16) and (17). Then $\varphi^{*} \in \mathcal{M}$ is a critical point of $\left.V_{I}\right|_{\mathcal{M}}$ (i.e. $\left.\nabla_{\varphi} V_{I}\right|_{\mathcal{M}}\left(\varphi^{*}\right)=0$ ), if and only if $B \varphi^{*}$ is a fixed point of the unperturbed (i.e. $\tilde{\omega}=0$ ) incremental dynamics (5).

At the light of Lemma 2, we can state the following lemma, which recovers the result of [14, Proposition 3.3.2] in terms of the incremental dynamics of the system. It states that, for a symmetric interconnection topology, any zero-grounded input preserves the phase-locking.

Lemma 3 (Almost global asymptotic phase-locking) Let $\varpi: \mathbb{R}_{\geq 0} \rightarrow \mathbb{R}^{N}$ be any signal satisfying $\tilde{\omega}(t)=0$, for all $t \geq 0$, where $\tilde{\omega}$ is defined in (4). If the interconnection matrix $k \in \mathbb{R}_{\geq 0}^{N \times N}$ is symmetric, then almost all trajectories of (1) converge to a stable phase-locked solution.

We stress that Lemma 3 is an almost global result. In particular, it follows from the fact that $V_{I}$ is striclty decreasing along the trajectories of (5) when no inputs are applied (cf. Claim 1), if and only if the state does not belong to the critical set of $\left.V_{I}\right|_{\mathcal{M}}$. From Lemma 2, this set corresponds to the fixed points of the incremental dynamics. Hence, almost all trajectories converge to the set of local minima of $\left.V_{I}\right|_{\mathcal{M}}$, that is to stable phase-locked solutions. The precise proof is omitted here.

Remark 2 (Lyapunov characterization of robust 0-AS phase-locked solution) In view of Definition 1 and Lemma 3 , the set of isolated local minima of $\left.V_{I}\right|_{\mathcal{M}}$ defines 0AS phase-locked solutions of (1). This set can be easily computed by means of Lemma 1, and its robustness property follows directly from Theorem 1 . In particular the set $\mathcal{O}_{k}$ of Definition 1, which describes robust phase-locked solutions in terms of the incremental dynamics, is non-empty provided that the function $\left.V_{I}\right|_{\mathcal{M}}$ has at least one isolated local minimum.

As stressed by Lemma 1 , the origin of $\mathcal{M}$ is always a local minimum of $\left.V_{I}\right|_{\mathcal{M}}$. This particular phase-locked solution corresponds to the synchronized state, that is the phaselocked state characterized by zero phase differences between each couple of oscillators (i.e. $\tilde{\theta}=0$, cf. [8], [20], [6], [14] and reference therein). The following corollary exploits the incremental Lyapunov analysis to extend known results on the robustness of the synchronized state to external inputs. Its proof directly follows from Theorem 1 and Lemma 1, and is therefore omitted.

Corollary 2 (LISS of the synchronized state) If the interconnection matrix $k \in \mathbb{R}_{\geq 0}^{N \times N}$ is symmetric, and the origin 
of $\mathcal{M}$ is an isolated critical point of $\left.V_{I}\right|_{\mathcal{M}}$, then the origin of $\mathcal{M}$ is LISS with respect to small inputs.

In [18, Theorem 2] sufficient conditions are given for the existence of an asymptotically stable synchronized state for the Kuramoto system with general bidirectional (i.e. symmetric) interconnection and constant different natural frequencies in terms of the algebraic properties of the coupling graph. The proof of Corollary 2 gives an extension of that result to time-varying inputs, by providing sufficient conditions on the coupling strength $K$, introduced in (13), for the local ISS of the synchronized state (i.e. the origin of the incremental dynamics). Indeed, Equation (29) in the proof can be inverted to give

$$
K>\frac{2 \delta_{\omega}}{\sigma\left(\frac{\delta}{2}\right)},
$$

where $\delta_{\omega}$ denotes the inputs amplitude, $\delta$ is the minimum distance between two critical sets of the unperturbed incremental dynamics, and $\sigma$ is a $\mathcal{K}_{\infty}$ function. We stress that both $\delta$ and $\sigma$ depend only on the normalized interconnection matrix $E$ introduced in (13) and are therefore independent of the coupling strength $K$. It follows that a larger input $\tilde{\omega}$ can be tolerated if $K$ is sufficiently large.

In the following corollary we formally state this reasoning by considering the case when the amplitude of the inputs (including different natural frequencies, unmodeled dynamics, mean-field feedback, etc.) is bounded and the coupling strength $K$ is seen as a tunable gain. In this situation, the system is practically stable in the sense of [39]. That is, given any precision $d>0$, we can find a sufficiently large coupling strength $K$ that makes the solutions of (1) phaselocked, modulo this prescribed tolerance $d$. The proof comes directly from [39] in view of Claim 2, and recalling that the Lyapunov function $V_{I}$ does not depend on the tuning parameter $K$.

Corollary 3 (Practical phase-locking) Consider any symmetric interconnection matrix $k \in \mathbb{R}_{\geq 0}^{N \times N}$. Let $\mathcal{O}_{k}$ be defined as in Definition 1 and suppose that it is non-empty. Then for any $\delta_{\omega}>0$ and any $d>0$ there exist $K>0$, a neighborhood $\mathcal{P}$ of $\mathcal{O}_{k}$, and a class $\mathcal{K} \mathcal{L}$ function $\beta$, such that, for all $\tilde{\theta}_{0} \in \mathcal{P}$ and all $\|\tilde{\omega}\| \leq \delta_{\omega}$, the trajectory of (5) satisfies

$$
|\tilde{\theta}(t)|_{\mathcal{O}_{k}} \leq \beta\left(\left|\tilde{\theta}_{0}\right|_{\mathcal{O}_{k}}, t\right)+d, \quad \forall t \geq 0 .
$$

The above result states that it is possible to arbitrarily tune the steady state value of the phase differences by choosing a convenient coupling strength $K$. The proposed Lyapunov function permits to exploit Lyapunov-based practical stability analysis also for the asymptotically stable fixed points, other than synchronization (i.e. $\tilde{\theta}^{*} \neq 0$ ), of the unperturbed Kuramoto system, which may appear for interconnection topologies different from the complete graph [14, Section 3.4]. Even if the notation may be confusing, we stress that the set $\mathcal{O}_{k}$ does not depend on the coupling strength $K$, but only on the normalized interconnection matrix $E$ defined in (13).
In the case of all-to-all coupling this result partially extends [15, Section IV] to time-varying (bounded) naturalfrequencies. Indeed in this case the same arguments of our proof apply locally (i.e. for $\tilde{\theta}$ inside the half circle) to the quadratic Lyapunov functions presented in [15] which can indeed be seen as a small-angles approximation of the one proposed here (see also Proposition 1 above).

In the case of all-to-all coupling and constant different natural frequencies, necessary and sufficient conditions for the existence of an asymptotically stable perturbed synchronized state and its explicit expression are given in [17]. Again, Corollary 2 and Corollary 3 partially generalize this result by giving sufficient conditions for the locall ISS of the synchronized state and by giving an estimate on the size of the phase differences.

\section{PROOFS}

\section{A. Proof of Theorem 1}

In order to develop our robustness analysis we first introduce a suitable Lyapunov function

$$
V_{I}(\tilde{\theta})=2 \sum_{i=1}^{N} \sum_{j=1}^{N} E_{i j}\left(\sin \left(\frac{\theta_{i}-\theta_{j}}{2}\right)\right)^{2}
$$

where the incremental variable $\tilde{\theta}$ is defined in (6), and the normalized interconnection matrix $E$ in (13). Then we compute the derivative of $V_{I}$ along the trajectories of the incremental dynamics (5)

$$
\dot{V}_{I}(\tilde{\theta})=\left(\nabla_{\tilde{\theta}} V_{I}\right)^{T} \dot{\tilde{\theta}}
$$

We thus have the following lemma which gives a useful form of $\dot{V}_{I}$.

Claim 1 If $k$ is symmetric, then $\dot{V}_{I}=-2\left(K \chi^{T} \chi+\chi^{T} \tilde{\omega}\right)$, where $\chi(\tilde{\theta}):=\nabla_{\theta} V(\tilde{\theta})=\left[\sum_{j=1}^{N} E_{i j} \sin \left(\theta_{j}-\theta_{i}\right)\right]_{i=1, \ldots, N}$.

From Claim 1, we see that if the inputs are small, there are regions of the phase space where the derivative of $V_{I}$ is negative even in the presence of perturbations. More precisely, it holds that:

$$
|\chi| \geq \frac{2|\tilde{\omega}|}{K} \Rightarrow \dot{V}_{I} \leq-K \chi^{T} \chi
$$

Let $\mathcal{F}$ be the set of critical points of $\left.V_{I}\right|_{\mathcal{M}}$ (i.e. $\mathcal{F}=$ $\left.\left\{\varphi^{*} \in \mathcal{M}:\left.\nabla_{\varphi} V_{I}\right|_{\mathcal{M}}\left(\varphi^{*}\right)=0\right\}\right)$, where $\mathcal{M}$ is defined in (16) and $\left.V_{I}\right|_{\mathcal{M}}$ in (14). Then, from Lemma 1 and recalling that $\chi=\nabla_{\theta} V$, it results that $|\chi|=0$ if and only if $\tilde{\theta} \in \mathcal{F}$. Since $|\chi|$ is a positive definite function of $\tilde{\theta}$ on a compact set, for [40, Lemma 4.3], there exists a $\mathcal{K}_{\infty}$ function $\sigma$ such that

$$
|\chi| \geq \sigma\left(|\tilde{\theta}|_{\mathcal{F}}\right)
$$

Let $\mathcal{U}:=\mathcal{F} \backslash \mathcal{O}_{k}$. In view of Lemma $2, \mathcal{U}$ denotes the set of all the critical points of $\left.V_{I}\right|_{\mathcal{M}}$ which are not asymptotically stable fixed points of the incremental dynamics. Since $\left.\nabla V_{I}\right|_{\mathcal{M}}$ is a Lipschitz function on a compact space, it can be different from zero only on a finite collection of open sets. 
That is $\mathcal{U}$ and $\mathcal{O}_{k}$ can be expressed as the disjoint union of a finite family of closed sets

$$
\mathcal{U}=\bigcup_{i \in I_{\mathcal{U}}} \nu_{i}, \quad \mathcal{O}_{k}=\bigcup_{i \in I_{\mathcal{O}_{k}}}\left\{\phi_{i}\right\}
$$

where $I_{\mathcal{U}}, I_{\mathcal{O}_{k}} \subset \mathbb{N}$ are finite sets, $\left\{\nu_{i}, i \in I_{\mathcal{U}}\right\}$ is a family of closed subsets of $\mathcal{M}$, and $\left\{\left\{\phi_{i}\right\}, i \in I_{\mathcal{O}_{k}}\right\}$ is a family of singletons of $\mathcal{M}$, such that $a \neq b$ implies $a \cap b=\emptyset$ for any $a, b \in\left\{\nu_{i}, i \in I_{\mathcal{U}}\right\} \bigcup\left\{\left\{\phi_{i}\right\}, i \in I_{\mathcal{O}_{k}}\right\}=: \mathcal{F}_{S}$. Define

$$
\delta:=\min _{a, b \in \mathcal{F}_{S}, a \neq b} \inf _{\tilde{\theta} \in a}|\tilde{\theta}|_{b}
$$

which represents the minimum distance between two critical sets, and, at the light of Lemma 2, gives a geometric characterization of the fixed points of the incremental dynamics (1). Note that, since $\mathcal{F}_{S}$ is finite, $\delta>0$. Define

$$
\delta_{\omega}^{\prime}=\frac{K}{2} \sigma\left(\frac{\delta}{2}\right)
$$

and let

$$
\delta_{\tilde{\theta}}:=\frac{\delta}{2}
$$

which gives an estimate of the size of the region of attraction, modulo the shape of the level sets of the Lyapunov function $V_{I}$. Then the following claim holds true.

Claim 2 For all $i \in I_{\mathcal{O}_{k}}$, all $\tilde{\theta} \in \mathcal{B}\left(\phi_{i}, \delta_{\tilde{\theta}}\right)$, and all $|\tilde{\omega}| \leq \delta_{\omega}^{\prime}$, it holds that

$$
\left|\tilde{\theta}-\phi_{i}\right| \geq \sigma^{-1}\left(\frac{2|\tilde{\omega}|}{K}\right) \Rightarrow \dot{V}_{I} \leq-K \sigma^{2}\left(\left|\tilde{\theta}-\phi_{i}\right|\right) .
$$

From [40, Lemma 4.3], since for all $i \in I_{\mathcal{O}_{k}}$ the function $V_{I}(\tilde{\theta})-V_{I}\left(\phi_{i}\right)$ is positive definite on $\mathcal{B}\left(\phi_{i}, \delta_{\tilde{\theta}}\right)$, and $\mathcal{B}\left(\phi_{i}, \delta_{\tilde{\theta}}\right)$ is compact, there exists $\mathcal{K}_{\infty}$ functions $\underline{\alpha}_{i}, \bar{\alpha}_{i}$ such that, $\forall \tilde{\theta} \in$ $\mathcal{B}\left(\phi_{i}, \delta_{\tilde{\theta}}\right)$,

$$
\underline{\alpha}_{i}\left(\left|\tilde{\theta}-\phi_{i}\right|\right) \leq V_{I}(\tilde{\theta})-V_{I}\left(\phi_{i}\right) \leq \bar{\alpha}_{i}\left(\left|\tilde{\theta}-\phi_{i}\right|\right) .
$$

Define

$$
\underline{\alpha}:=\min _{i \in I_{\mathcal{O}_{k}}} \underline{\alpha}_{i}, \bar{\alpha}:=\max _{i \in I_{\mathcal{O}_{k}}} \bar{\alpha}_{i},
$$

that is, for all $i \in I_{\tilde{O}}$ and all $\tilde{\theta} \in \mathcal{B}\left(\phi_{i}, \delta_{\tilde{\theta}}\right)$, it results that $\underline{\alpha}\left(\left|\tilde{\theta}-\phi_{i}\right|\right) \leq V_{I}(\tilde{\theta})-V_{I}\left(\phi_{i}\right) \leq \bar{\alpha}\left(\left|\tilde{\theta}-\phi_{i}\right|\right)$. An estimates of the ISS gain is then given by

$$
\rho(s):=\underline{\alpha}^{-1} \circ \bar{\alpha} \circ \sigma^{-1}\left(\frac{2}{K} s\right),
$$

where and $\sigma$ is defined in (21). The tolerated input bound is given by

$$
\delta_{\omega}:=\rho^{-1}\left(\delta_{\tilde{\theta}}\right) \leq \delta_{\omega}^{\prime} .
$$

From [41, Section 10.4] and Claim 2, it follows that, for all $\|\tilde{\omega}\| \leq \delta_{\omega}$, the set $\mathcal{B}\left(\mathcal{O}_{k}, \delta_{\tilde{\theta}}\right)$ is forward invariant for the system (5) . Invoking [42] and [41, Section 10.4], Claim 2 thus implies that there exists a class $\mathcal{K} \mathcal{L}$ function $\beta$ such that, for all $\|\tilde{\omega}\| \leq \delta_{\omega}$, and all $\tilde{\theta}_{0} \in \mathcal{B}\left(\mathcal{O}_{k}, \delta_{\tilde{\theta}}\right)$, the trajectory of (5) satisfies $|\tilde{\theta}(t)| \leq \beta\left(\left|\tilde{\theta}_{0}\right|, t\right)+\rho(\|\tilde{\omega}\|)$, for all $t \geq 0$.
Proof of Claim 1 Consider the derivative of the incremental Lyapuonv function $V_{I}$, defined in (14), along the trajectories of the incremental dynamics (5):

$$
\begin{gathered}
\dot{V}_{I}(\tilde{\theta}):=\left(\nabla_{\tilde{\theta}} V_{I}\right)^{T} \dot{\tilde{\theta}}=\sum_{i, j=1}^{N} E_{i j} \sin \left(\theta_{j}-\theta_{i}\right)\left(\dot{\theta}_{j}-\dot{\theta}_{i}\right)= \\
-2 \sum_{i, j=1}^{N} E_{i j} \sin \left(\theta_{j}-\theta_{i}\right) \dot{\theta}_{i},
\end{gathered}
$$

where the last inequality comes from the fact that, if $E$ is a symmetric matrix, then $\sum_{i, j=1}^{N} E_{i j} \sin \left(\theta_{j}-\theta_{i}\right) \dot{\theta}_{j}=$ $-\sum_{i, j=1}^{N} E_{i j} \sin \left(\theta_{j}-\theta_{i}\right) \dot{\theta}_{i}$. For the same reason it holds that $\bar{\omega} \sum_{i, j=1}^{N} E_{i j} \sin \left(\theta_{j}-\theta_{i}\right)=0$. Since, from (1), $\dot{\theta}_{i}=$ $\bar{\omega}+\tilde{\omega}_{i}+K \sum_{l=1}^{N} E_{i l} \sin \left(\theta_{l}-\theta_{i}\right)$, we get that

$\dot{V}_{I}=-2 \sum_{i=1}^{N}\left(\sum_{i=1}^{N} E_{i j} \sin \left(\theta_{j}-\theta_{i}\right)\right)\left(K \sum_{l=1}^{N} E_{i l} \sin \left(\theta_{l}-\theta_{i}\right)+\tilde{\omega}_{i}\right)$,

which proves the claim.

Proof of Claim 2 From Claim 1 it holds that $\dot{V}_{I}=$ $-2 K|\chi|^{2}-2 \chi^{T} \tilde{\omega} \leq-2 K|\chi|^{2}-2|\chi||\tilde{\omega}|$. That is

$$
|\chi| \geq \frac{2|\tilde{\omega}|}{K}, \quad \Rightarrow \quad \dot{V}_{I} \leq-K|\chi|^{2} .
$$

Since $|\tilde{\omega}| \leq \delta_{\omega}^{\prime}$ implies, for (24)-(25), $\sigma^{-1}\left(\frac{2|\tilde{\omega}|}{K}\right) \leq \delta_{\tilde{\theta}}$, and, for all $\tilde{\theta} \in \mathcal{B}\left(\phi_{i}, \delta_{\tilde{\theta}}\right),|\tilde{\theta}|_{\mathcal{F}}=\left|\tilde{\theta}-\phi_{i}\right|$, it results that

$$
\left|\tilde{\theta}-\phi_{i}\right| \geq \sigma^{-1}\left(\frac{2|\tilde{\omega}|}{K}\right) \Rightarrow|\chi| \geq \frac{2|\tilde{\omega}|}{K},
$$

and, for $(21),-|\chi|^{2} \leq-\sigma^{2}\left(\left|\tilde{\theta}-\phi_{i}\right|\right)$. That is

$$
\left|\tilde{\theta}-\phi_{i}\right| \geq \sigma^{-1}\left(\frac{2|\tilde{\omega}|}{K}\right) \quad \Rightarrow \quad \dot{V}_{I} \leq-K \sigma^{2}\left(\left|\tilde{\theta}-\phi_{i}\right|\right) . \square
$$

\section{B. Proof of Proposition 1}

Input-to-State Gain: We start by computing the ISS gain $\rho$, defined in (28), in the particular case of all-to-all coupling. The first step is to compute the function $\sigma$, defined in (21), with respect to the origin of the incremental dynamics. That is we have to find a class $\mathcal{K}_{\infty}$ function $\sigma$, such that $|\chi(\tilde{\theta})| \geq$ $\sigma(|\tilde{\theta}|)$ for all $\tilde{\theta}$ in some neighborhood of the origin of the incremental dynamics, where $\chi$ is defined in Claim 1. The following claim gives an explicit expression of this function on the set $\mathcal{D}_{0}=\left\{\tilde{\theta}: \max _{i, j=1, \ldots, N}\left|\theta_{i}-\theta_{j}\right| \leq \frac{\pi}{2}\right\}$

Claim 3 In the case of all-to-all coupling, the function $\chi$ defined in Claim 1 satisfies, for any $\tilde{\theta} \in \mathcal{D}_{0},|\chi(\tilde{\theta})| \geq \frac{|\tilde{\theta}|}{\pi}$, that is $\sigma(r)=\frac{r}{\pi}$.

At the light of Claim 3, the ISS gain $\rho$ can be easily computed through (28). Indeed, in the all-to-all case, the Lyapunov function $V_{I}$, provided in (14), becomes

$$
V_{I}(\tilde{\theta})=2 \sum_{i, j=1}^{N}\left(\sin \left(\frac{\theta_{i}-\theta_{j}}{2}\right)\right)^{2} \text {. }
$$


Using the fact that $z \geq \sin z \geq \frac{2}{\pi} z$, for all $0 \leq z \leq \frac{\pi}{2}$, it follows that, for all $\tilde{\theta} \in \mathcal{D}_{0}$,

$$
\frac{2}{\pi^{2}}|\tilde{\theta}|^{2} \leq V_{I}(\tilde{\theta}) \leq \frac{1}{2}|\tilde{\theta}|^{2}
$$

Recalling the definition of the upper $\bar{\alpha}$ and lower $\underline{\alpha}$ estimates of the Lyapunov function with respect the set of asymptotically stable fixed point (27), and that, in the all-to-all case, this set reduces to the origin, we can conclude that

$$
\underline{\alpha}(r)=\frac{2}{\pi^{2}} r^{2}, \quad \bar{\alpha}(r)=\frac{1}{2} r^{2} .
$$

In view of Claim 3, and (28), it follows that the ISS gain $\rho$ in the statement of Theorem 1, can be chose as

$$
\rho(r)=\frac{\pi^{2}}{K} r .
$$

Input bound and invariant set: For Claim 3, the ISS gain computed in the last section is valid as soon as $\tilde{\theta}$ belongs to $\mathcal{D}_{0}$. In the following we compute an input bound which guarantees that trajectories starting in $\mathcal{D}_{0}$ remain inside $\mathcal{D}_{0}$. For the sake of generality, we actually show the forward invariance of $\mathcal{D}_{\epsilon}$ for any $\epsilon \in\left[0, \frac{\pi}{2}\right]$. To that end, we start by the following technical claim.

Claim 4 Given any $0 \leq \delta \leq \pi$, the following holds true:

$$
|\tilde{\theta}| \leq \sqrt{N} \delta \Rightarrow \max _{i, j=1, \ldots, N}\left|\theta_{i}-\theta_{j}\right| \leq \delta .
$$

At the light of Claim 4, we can compute the input bound $\delta_{\omega}^{\epsilon}$ which lets $\mathcal{D}_{\epsilon}$ be invariant for the systems (5) by imposing $\rho\left(\delta_{\omega}^{\epsilon}\right)=\sqrt{N}\left(\frac{\pi}{2}-\epsilon\right)$, where $\rho(s)=\frac{\pi^{2}}{K} s$ is the ISS gain in the statement of Theorem 1, which gives

$$
\delta_{\omega}^{\epsilon}=\frac{K \sqrt{N}}{\pi^{2}}\left(\frac{\pi}{2}-\epsilon\right) .
$$

Exponential convergence and attractiveness of $\mathcal{D}_{\epsilon}$ : From Claims 2 and 3, and (30), it follows that, for all $|\tilde{\omega}| \leq \delta_{\omega}^{\epsilon}$, and all $\tilde{\theta} \in \mathcal{D}_{0}$,

$$
|\tilde{\theta}| \geq \frac{2 \pi}{K}|\tilde{\omega}| \Rightarrow \dot{V}_{I} \leq-\frac{K}{\pi^{2}}|\tilde{\theta}|^{2} \leq-\frac{2 K}{\pi^{2}} V_{I} .
$$

By (comparison) Lemma [40, Lemma 3.4] and as long as $\min _{0 \leq s \leq t}|\tilde{\theta}(s)| \geq \frac{2 \pi}{K}\|\tilde{\omega}\|$,

$$
V_{I}(\tilde{\theta}(t)) \leq V_{I}(\tilde{\theta}(0)) e^{-\frac{2 K}{\pi^{2}} t} \forall t \geq 0 .
$$

From (30), this also implies

$$
\min _{0 \leq s \leq t}|\tilde{\theta}(s)| \geq \frac{2 \pi}{K}\|\tilde{\omega}\| \Rightarrow|\tilde{\theta}(t)| \leq \frac{\pi}{2}|\tilde{\theta}(0)| e^{-\frac{K}{\pi^{2}} t}, \forall t \geq 0 .
$$

From standard input-to-state stability arguments (see for example [41, Section 10.4] and [40, Lemma 4.4 and Theorem 4.10]), it follows that the system is exponentially inputto-state stable, and in particular that for all $\|\tilde{\omega}\| \leq \delta_{\omega}^{\epsilon}$ and all $\tilde{\theta}_{0}$ in $\mathcal{D}_{0}$,

$$
|\tilde{\theta}(t)| \leq \frac{\pi}{2}\left|\tilde{\theta}_{0}\right| e^{-\frac{K}{\pi^{2}} t}+\frac{\pi^{2}}{K}\|\tilde{\omega}\|, \forall t \geq 0 .
$$

Noticing that, if $\|\tilde{\omega}\| \leq \delta_{\omega}^{\epsilon}$,

$$
|\tilde{\theta}(t)| \leq \frac{\pi}{2}\left|\tilde{\theta}_{0}\right| e^{-\frac{K}{\pi^{2}} t}+\sqrt{N}\left(\frac{\pi}{2}-\epsilon\right), \forall t \geq 0,
$$

Claim 4 implies the attractiveness of $\mathcal{D}_{\epsilon}$ for all $\|\tilde{\omega}\| \leq \delta_{\omega}^{\epsilon}$.

Proof of Claim 3: In the case of all-to-all coupling the vector $\chi \in \mathbb{R}^{N}$ is given by $\chi(\tilde{\theta})=\left[\sum_{j=1}^{N} \sin \left(\theta_{j}-\theta_{i}\right)\right]_{i=1, \ldots, N}$. First of all note that $|\chi| \geq \max _{i=1, \ldots, N}\left|\sum_{j=1}^{N} \sin \left(\theta_{j}-\theta_{i}\right)\right|$. For the sake of simplicity, renumber the oscillator phases indexes in such a way that $\theta_{i}<\theta_{j}$ whenever $i<j$. It then results that $\left|\sum_{j=1}^{N} \sin \left(\theta_{j}-\theta_{1}\right)\right|=\sum_{j=1}^{N} \sin \left|\theta_{j}-\theta_{1}\right|$, $\left|\sum_{j=1}^{N} \sin \left(\theta_{j}-\theta_{N}\right)\right|=\sum_{j=1}^{N} \sin \left|\theta_{j}-\theta_{N}\right|$, and, for any $i \notin\{1, N\}, \quad\left|\sum_{j=1}^{N} \sin \left(\theta_{j}-\theta_{i}\right)\right|=$ $\left|\sum_{1 \leq j<i} \sin \right| \theta_{j}-\theta_{i}\left|-\sum_{i<j \leq N} \sin \right| \theta_{j}-\theta_{i}||$. Recalling that for any $a, b>0,|a-b| \leq \max \{a, b\}$, and noticing that, for $\left|\theta_{i}-\theta_{j}\right| \leq \frac{\pi}{2}, \sum_{1 \leq j<i} \sin \left|\theta_{j}-\theta_{i}\right|<\sum_{j=1}^{N} \sin \left|\theta_{j}-\theta_{N}\right|$ and $\sum_{i<j \leq N} \sin \left|\theta_{j}-\theta_{i}\right|<\sum_{j=1}^{N} \sin \left|\theta_{j}-\theta_{1}\right|$, we can conclude that $\max _{i=1, \ldots, N}\left|\sum_{j=1}^{N} \sin \left(\theta_{j}-\theta_{i}\right)\right|=$ $\max \left\{\sum_{j=1}^{N} \sin \left|\theta_{j}-\theta_{1}\right|, \sum_{j=1}^{N} \sin \left|\theta_{j}-\theta_{N}\right|\right\}$.

Since we are looking for a lower bound to $\max _{i=1, \ldots, N}\left|\sum_{j=1}^{N} \sin \left(\theta_{j}-\theta_{i}\right)\right|$ in term of $|\tilde{\theta}|$, we have to find which $\tilde{\theta}$ minimizes it for a given $|\tilde{\theta}|$. In particular, we are now going show that this alway happens when $\sum_{j=1}^{N} \sin \left|\theta_{j}-\theta_{1}\right|=\sum_{j=1}^{N} \sin \left|\theta_{j}-\theta_{N}\right|$. Define $\tilde{\theta}_{\infty}:=\theta_{N}-\theta_{1} \leq \frac{\pi}{2}, x_{i}:=\theta_{i+1}-\theta_{1}$, for all $i=1, \ldots, N-2$, and $x:=\left[x_{i}\right]_{i=1, \ldots, N-2} \in\left[0, \tilde{\theta}_{\infty}\right]^{N-2}$. Then it results that $\sum_{j=1}^{N} \sin \left|\theta_{j}-\theta_{1}\right|=\sin \tilde{\theta}_{\infty}+\sum_{i=1}^{N-2} \sin x_{i}=: a(x)$, and $\sum_{j=1}^{N} \sin \left|\theta_{j}-\theta_{N}\right|=\sin \tilde{\theta}_{\infty}+\sum_{i=1}^{N-2} \sin \left(\tilde{\theta}_{\infty}-x_{i}\right):=b(x)$. Define $A=\left\{x \in\left[0, \tilde{\theta}_{\infty}\right]^{N-2}: a(x)=b(x)\right\}$. Hence, we want to show that $\operatorname{argmin}_{x} \max \{a(x), b(x)\} \in A$. To show this note that the only local minimum of $a(x)$ is $x^{*}=0$, and that the only local minimum of $b(x)$ is $x^{*}=\tilde{\theta}_{\infty} \mathbf{1}$. Now, since $0 \notin\{x: a(x)>b(x)\}$ and $\tilde{\theta}_{\infty} \mathbf{1} \notin\{x: a(x)<b(x)\}$, the minimum of $\max \{a(x), b(x)\}$, it it exists, belongs to $A$. The minimum exists since $\max \{a(x), b(x)\}$ is a continuous function on a compact set. Hence we have to minimize the function $a(x)$ on $\left[0, \tilde{\theta}_{\infty}\right]$, under the constraint $a(x)-b(x)=0$, that is on $\left[0, \tilde{\theta}_{\infty}\right]^{N-2} \cap A$. In particular we have look for its critical points under this constraint. Since $a(x)$ and the constrained function $a(x)-b(x)$ are smooth functions, we can do this by applying the method of Lagrange multipliers (see Appendix). That is by solving the equation $\nabla_{(x, \lambda)} \Lambda(x, \lambda)=0$, where $\Lambda(x, \lambda)=a(x)-\lambda(a(x)-b(x))$. Differentiating with respect to $x_{i}$ gives $\cos x_{i}-\lambda\left(\cos x_{i}+\cos \left(\tilde{\theta}-x_{i}\right)\right)=0$, which implies $\cos x_{i}=C_{\lambda}$, for all $i=1, \ldots, N-2$ and some constant $C_{\lambda}$. Differentiating with respect to $\lambda$ gives, by posing $\cos x_{i}=C_{\lambda}$ and $\sin x_{i}=\sqrt{1-C_{\lambda}^{2}}$, and using the trigonometric identity $\sin (c-d)=\sin c \cos d-\cos c \sin d$, $(N-2) \sqrt{1-C_{\lambda}^{2}}-(N-2)\left(\sin \tilde{\theta}_{\infty} C_{\lambda}-\cos \tilde{\theta}_{\infty} \sqrt{1-C_{\lambda}^{2}}\right)=$ 0 . On the considered domain, this equation admits only one solution $C_{\lambda}=\cos \frac{\tilde{\theta}_{\infty}}{2}$. Since $C_{\lambda}=\cos x_{i}$, it follows that $\cos \frac{\tilde{\theta}_{\infty}}{2}=\cos x_{i}=\cos \left(\tilde{\theta}_{\infty}-x_{i}\right)$, which is compatible 
with $\cos x_{i}-\lambda\left(\cos x_{i}+\cos \left(\tilde{\theta}_{\infty}-x_{i}\right)\right)=0$, by putting $\lambda=\frac{1}{2}$. That is $a(x)$ has only one critical point $x^{*}=\frac{\tilde{\theta}_{\infty}}{2} \mathbf{1}$ on $\left[0, \tilde{\theta}_{\infty}\right]^{N-2} \cap A$. Define, for some $0<\epsilon \leq \frac{\tilde{\theta}_{\infty}}{4}$ and $p, q \in\{1, \ldots, N-2\}, p \neq q$, the vector $x^{\epsilon}$ in the following way: $x_{i}^{\epsilon}:=x_{i}^{*}$ if $i \notin\{p, q\}$ and $x_{p}^{\epsilon}:=x_{p}^{*}-\epsilon$, $x_{q}^{\epsilon}:=x_{p}^{*}+\epsilon$. Note that $x^{\epsilon} \in A$ and that $a\left(x^{*}\right)-a\left(x^{\epsilon}\right)=$ $2 \sin \left(\tilde{\theta}_{\infty} / 2\right)-\left(\sin \left(\tilde{\theta}_{\infty} / 2-\epsilon\right)+\sin \left(\tilde{\theta}_{\infty} / 2+\epsilon\right)\right)<0$, where the last inequalities comes from the fact that the sine function is concave on the considered domain, which implies that $x^{*}$ is a maximum of $a(x)$ on $\left[0, \tilde{\theta}_{\infty}\right]^{N-2} \cap A$. Since $a(x)$ is a continuous function and $\left[0, \tilde{\theta}_{\infty}\right]^{N-2} \cap A$ is compact, $a(x)$ has a minimum on $\left[0, \tilde{\theta}_{\infty}\right]^{N-2} \cap A$. The observation that $x^{*}$ is the only critical point of $a(x)$ in the interior of $\left[0, \tilde{\theta}_{\infty}\right]^{N-2} \cap A$, implies that the minimum belongs to the border of the set $\left[0, \tilde{\theta}_{\infty}\right]^{N-2} \cap A$. If $N-2$ is even, this border is given by the points where $(N-2) / 2$ of the $x_{i}$ are zero and the other $(N-2) / 2$ are equal to $\theta_{\infty}$. If $N-2$ is odd, this border is given by the points where $(N-3) / 2$ of the $x_{i}$ are zero, one of them is equal to $\tilde{\theta}_{\infty} / 2$ and the other $(N-3) / 2$ are equal to $\tilde{\theta}_{\infty}$. In both case it results that, on $\left[0, \tilde{\theta}_{\infty}\right]^{N-2} \cap A, a(x) \geq \frac{N}{2} \sin \tilde{\theta}_{\infty}$. Finally, we can conclude that $|\chi| \geq \frac{N}{2} \sin \left(\max _{i, j=1, \ldots, N}\left|\theta_{i}-\theta_{j}\right|\right)=\frac{N}{2} \sin \tilde{\theta}_{\infty}$. By recalling that $\sin z \geq \frac{2}{\pi} z$, for all $0 \leq z \leq \frac{\pi}{2}$, and that $\tilde{\theta}_{\infty}=\max _{i, j=1, \ldots, N}\left|\theta_{i}-\theta_{j}\right| \geq \frac{|\tilde{\theta}|}{N-1}$, we can conclude that, for all $\tilde{\theta} \in \mathcal{D}_{0},|\chi(\tilde{\theta})| \geq \frac{N}{\pi(N-1)}|\tilde{\theta}| \geq \frac{|\tilde{\theta}|}{\pi}$, which proves the claim.

Proof of Claim 4: We want to minimize the Euclidean norm $|\tilde{\theta}|$, with the constraint that $\max _{i, j=1, \ldots, N}\left|\theta_{i}-\theta_{j}\right|=$ $\tilde{\theta}_{\infty}$. For the sake of simplicity, renumber the oscillator phases indexes in such a way that $\theta_{i}<\theta_{j}$ whenever $i<j$. The problem can then be translated in minimizing $|\tilde{\theta}|$, with the constraint that $\theta_{N}-\theta_{1}=\tilde{\theta}_{\infty}$. Since the Euclidean norm and the constrained function are smooth, we can apply the method of Lagrange multipliers (see Appendix). That is, we can find critical points of $|\tilde{\theta}|$, under the constraint $\theta_{N}-\theta_{1}=\tilde{\theta}_{\infty}$, by solving the equation $\nabla_{(\theta, \lambda)}\left(\sum_{i, j=1}^{N}\left(\theta_{i}-\theta_{j}\right)^{2}-\lambda\left(\theta_{N}-\theta_{1}-\tilde{\theta}_{\infty}\right)\right)=0$. Differentiating with respect to $\theta_{l}$, for $l \notin\{1, N\}$, gives

$$
\sum_{j=1}^{N}\left(\theta_{l}-\theta_{j}\right)=0 .
$$

Differentiating with respect to $\lambda$ just gives the constraint $\theta_{N}-\theta_{1}=\tilde{\theta}_{\infty}$. Differentiating with respect to $\theta_{1}$ gives $4 \sum_{j=1}^{N}\left(\theta_{1}-\theta_{j}\right)+\lambda=0$, differentiating with respect to $\theta_{N}$ gives $4 \sum_{j=1}^{N}\left(\theta_{N}-\theta_{j}\right)-\lambda=0$, and, by solving with respect to $\lambda$ and using the constraint, we get

$$
\sum_{j=1}^{N}\left(\theta_{1}-\theta_{j}\right)+\sum_{j=1}^{N}\left(\theta_{N}-\theta_{j}\right)=0 .
$$

Equations (31) and (32) admit a solution $\theta_{i}^{*}-\theta_{j}^{*}=$ 0 for all $(i, j) \notin\{(1, N),(N, 1)\}$, and $\theta_{i}^{*}-\theta_{1}^{*}=$ $\frac{\tilde{\theta}_{\infty}}{2}, \theta_{i}^{*}-\theta_{N}^{*}=-\frac{\tilde{\theta}_{\infty}}{2}$, for all $i \notin\{1, N\}$. By convexity of the quadratic function it is easy to show that this point correspond to a minimum. To show the uniqueness of this critical point, note that the equation $\nabla_{\theta, \lambda}\left(\sum_{i, j=1}^{N}\left(\theta_{i}-\theta_{j}\right)^{2}-\lambda\left(\theta_{N}-\theta_{1}-\tilde{\theta}_{\infty}\right)\right)=0$ can be rewritten in terms of the linear system

$$
\begin{gathered}
A\left(\begin{array}{c}
\theta_{1} \\
\theta_{2} \\
\vdots \\
\theta_{N-1} \\
\theta_{N}
\end{array}\right)=\left(\begin{array}{c}
-\frac{\lambda}{4} \\
0 \\
\vdots \\
0 \\
\frac{\lambda}{4}
\end{array}\right), \\
\lambda=\tilde{\theta}^{\infty},
\end{gathered}
$$

where the matrix $A \in \mathbb{R}^{N \times N}$, defined as

$$
A:=\left(\begin{array}{ccccc}
N-1 & -1 & \ldots & -1 & -1 \\
-1 & N-1 & \ldots & -1 & -1 \\
\vdots & \vdots & \ddots & \vdots & \vdots \\
-1 & -1 & \ldots & N-1 & -1 \\
-1 & -1 & \ldots & -1 & N-1
\end{array}\right),
$$

has full rank since it is diagonally dominant with strictly positive diagonal entries. We can then conclude that, if $\max _{i, j=1, \ldots, N}\left|\theta_{i}-\theta_{j}\right|=\theta_{\infty}$, then

$$
\begin{gathered}
|\tilde{\theta}|^{2}= \\
\sum_{i, j=1}^{N}\left(\theta_{i}-\theta_{j}\right)^{2} \geq 2 \tilde{\theta}_{\infty}^{2}+2 \sum_{j=2}^{N-2} \frac{\tilde{\theta}_{\infty}^{2}}{4}+2 \sum_{j=2}^{N-2} \frac{\tilde{\theta}_{\infty}^{2}}{4}=N \tilde{\theta}_{\infty}^{2} .
\end{gathered}
$$

This implies that, if $|\tilde{\theta}| \leq \sqrt{N} \delta$, then $\max _{i, j=1, \ldots, N} \mid \theta_{i}-$ $\theta_{j} \mid \leq \delta$, for any $0 \leq \delta \leq \pi$.

\section{Proof of Corollary 1}

The Corollary is a trivial consequence of Theorem 1 by noting that, since $\left|\left(\tilde{I}_{M F}\right)_{i}\right|=\left|\left(I_{M F}\right)_{i}-\frac{1}{N} \sum_{j}\left(I_{M F}\right)_{j}\right| \leq$ $\max _{j}\left|\left(I_{M F}\right)_{j}\right|$ and $\left|\left(I_{M F}\right)_{i}\right|<2 N \bar{\gamma}$, for all $i=1, \ldots, N$, it results that $\left|\tilde{I}_{M F}\right|<2 \bar{\gamma} \sqrt{N} N$. By letting $\delta_{\omega}$ be defined as in (29), from Theorem 1, the system is LISS, provided that $\left|\omega^{\perp}\right|+\left|\tilde{I}_{M F}\right|<\left|\omega^{\perp}\right|+2 \bar{\gamma} \sqrt{N} N \leq \delta_{\omega}$.

\section{Proof of Lemma 1}

By the definition (19) of $V(\theta)$, it holds that $\nabla_{\theta} V(\theta)=$ $\left.\nabla_{\theta} V_{I}\right|_{\mathcal{M}}(A \theta)=\left.A^{T} \nabla_{A \theta} V_{I}\right|_{\mathcal{M}}(A \theta)$. Hence

$$
\left.\nabla_{A \theta} V_{I}\right|_{\mathcal{M}}(A \theta)=0 \Rightarrow \nabla_{\theta} V(\theta)=0,
$$

by the linearity of $A^{T}$. Recalling that, since $\operatorname{rank} A=N-1$, $\operatorname{ker} A^{T}=0$, it follows that

$$
\nabla_{\theta} V(\theta)=\left.0 \quad \Rightarrow \quad \nabla_{A \theta} V_{I}\right|_{\mathcal{M}}(A \theta)=0,
$$

which proofs the first part of the lemma.

To proove the second part of the lemma, we note that if $\theta^{*}$ is a local minimum of $V$ then there exists a neighbourhood $U$ of $\theta^{*}$ such that $V(\theta) \geq V\left(\theta^{*}\right)$ for all $\theta \in U$. That is, $\left.V_{I}\right|_{\mathcal{M}}(A \theta) \geq\left. V_{I}\right|_{\mathcal{M}}\left(A \theta^{*}\right)$ for all $\theta \in U$. That is $\left.V_{I}\right|_{\mathcal{M}}(\varphi) \geq$ $\left.V_{I}\right|_{\mathcal{M}}\left(\varphi^{*}\right)$ for all $\varphi \in W=A U$, where $\varphi^{*}=A \theta^{*}$. A similar proof holds for maxima.

The third part of the lemma follows from the fact the function $\left.V_{I}\right|_{\mathcal{M}}$ is positive definite and $\left.V_{I}\right|_{\mathcal{M}}(0)=0$. 


\section{E. Proof of Lemma 2}

From Lemma 1 , it results that $\phi^{*} \in \mathcal{M}$ is a critical point of $\left.V_{I}\right|_{\mathcal{M}}$ if and only if $\theta^{*} \in \mathrm{T}^{N}$ is a critical point of $V$, where $\phi^{*}=A \theta^{*}$, and $A$ is defined in (18). Moreover, when $\tilde{\omega}=0$, it results that the incremental dynamics (5) can be re-written as

$$
\dot{\theta}_{i}-\dot{\theta}_{j}=\chi_{j}(\theta)-\chi_{i}(\theta), \quad \forall i, j=1, \ldots, N,
$$

where $\chi(\theta)=\left[\chi_{i}(\theta)\right]_{i=1, \ldots, N}:=\nabla_{\theta} V(\theta)=$ $\sum_{j=1}^{N} E_{i j} \sin \left(\theta_{j}-\theta_{i}\right)$, and the normalized interconnection matrix $E$ is defined in (13). Hence, $\chi\left(\theta^{*}\right)=\nabla_{\theta} V\left(\theta^{*}\right)=0$ if and only if $\phi^{*}=A \theta^{*}$ is a critical point of $\left.V_{I}\right|_{\mathcal{M}}$; and $\chi_{j}\left(\theta^{*}\right)-\chi_{i}\left(\theta^{*}\right)=0$, for all $i, j=1, \ldots, N$, if and only if $B \phi^{*}=B A \theta^{*}$ is a fixed point of the unperturbed incremental dynamics, where $B$ is defined in (16). To prove the lemma it thus suffices to show that

$$
\chi\left(\theta^{*}\right)=0 \quad \Leftrightarrow \quad \chi_{j}\left(\theta^{*}\right)-\chi_{i}\left(\theta^{*}\right)=0, \quad \forall i, j=1, \ldots, N .
$$

One implication is straightforward: if $\chi\left(\theta^{*}\right)=0$, then in particular all of its components are zero, that is $\chi_{j}\left(\theta^{*}\right)-$ $\chi_{i}\left(\theta^{*}\right)=0$, for all $i, j=1, \ldots, N$. On the other hand, if $\chi_{j}\left(\theta^{*}\right)-\chi_{i}\left(\theta^{*}\right)=0$ for all $i, j=1, \ldots, N$, then there exists a constant $\bar{\chi}$, such that $\chi_{i}\left(\theta^{*}\right)=\bar{\chi}$ for all $i=1, \ldots, N$. Hence, it results that

$$
N \bar{\chi}=\sum_{i=1}^{N} \chi_{i}\left(\theta^{*}\right)=\sum_{i=1}^{N} \frac{\partial V}{\partial \theta_{i}}\left(\theta^{*}\right)=\sum_{i=1}^{N} \sum_{j=1}^{N} E_{i j} \sin \left(\theta_{j}^{*}-\theta_{i}^{*}\right) .
$$

Since the interconnection matrix $k$ is symmetric, so is the normalized interconnection matrix $E$ (cf. (13)), and it results that

$$
\sum_{i=1}^{N} \sum_{j=1}^{N} E_{i j} \sin \left(\theta_{j}^{*}-\theta_{i}^{*}\right)=0 .
$$

Consequently, $\chi_{i}\left(\theta^{*}\right)=\bar{\chi}=0$ for all $i=1, \ldots, N$, which proves the converse implication.

\section{APPENDIX}

An extremum of a continuously differentiable function $f$ : $\mathbb{R}^{n} \rightarrow \mathbb{R}$, under the constraints $g_{i}(x)=b_{i}, i=1, \ldots, m$, where $g_{i}: \mathbb{R}^{n} \rightarrow \mathbb{R}$ is continuously differentiable, and $b_{i} \in$ $\mathbb{R}$ belongs to the image of $g_{i}$, for all $i=1, \ldots, m$, can be found by constructing the Lagrangian function $F$ through the Lagrangian multipliers $\lambda_{i}, i=1, \ldots, m$,

$$
F\left(x, \lambda_{1}, \ldots, \lambda_{m}\right)=f(x)-\sum_{i=1}^{m} \lambda_{i}\left(g_{i}(x)-b_{i}\right)
$$

and by solving the set of equations

$$
\begin{gathered}
\frac{\partial}{\partial x_{i}} F\left(x, \lambda_{1}, \ldots, \lambda_{m}\right)=0, \\
\frac{\partial}{\partial \lambda_{j}} F\left(x, \lambda_{1}, \ldots, \lambda_{m}\right)=0,
\end{gathered}
$$

for all $i=1, \ldots, n$ and all $j=1, \ldots, m$. The optimal value $x^{*}$, is found together with the vector of Lagrangian multipliers $\lambda^{*}=\left(\lambda_{1}^{*}, \ldots, \lambda_{m}^{*}\right)$. See for example [43].

\section{REFERENCES}

[1] K. Wiesenfeld, P. Colet, and S. Strogatz, "Synchronization transitions in a disordered Josephson series array," Phys. Rev. Lett., vol. 76, 1996.

[2] — " "Frequency locking in Josephson arrays: Connection with the Kuramoto model," Physics Review E, vol. 57, 1998.

[3] W. Wang, Z. Kiss, and J. Hudson, "Clustering of arrays of chaotic chemical oscillators by feedback and forcing," Phys. Rev. Lett., vol. 86, 2001 .

[4] C. Peskin, Mathematical Aspects of Heart Phydiology. New-York: Courant Institute of Mathematical Sciences, 1975.

[5] A. Winfree, The Geometry of Biological Times. New-York: Springer, 1980.

[6] R. Sepulchre, D. A. Paley, and N. E. Leonard, "Stabilization of planar collective motion: All-to-all communication," IEEE Trans. on Automat. Contr., vol. 52, no. 5, pp. 811-824, May 2007.

[7] S. Strogatz, "From Kuramoto to Crawford: Exploring the onset of synchronization in population of coupled oscillators," Physica D, vol. 143, pp. 1-20, 2000.

[8] Y. Kuramoto, Chemical Oscillations, Waves, and Turbolence. Berlin: Springer, 1984.

[9] E. Izhikevich, "Which model to use for cortical spiking neurons?" IEEE Trans. on Neural Networks, vol. 15, pp. 1063-1070, 2004.

[10] - Dynamical Systems in Neuroscience: The Geometry of Excitability and Bursting. Cambridge, Mass: MIT Press, 2007.

[11] A. Pavlov, W. N. Victorovich, and H. Nijmeijer, Uniform Output Regulation of Nonlinear Systems. A Convergent Dynamics Approach. Birkhäuse, 2006.

[12] L. Scardovi, A. Sarlette, and R. Sepulchre, "Synchronization and balancing on the N-torus," Syst. \& Contr. Letters, vol. 56, no. 5, pp. 335-341, 2007.

[13] R. Olfati-Saber and R. M. Murray, "Consensus problems in networks of agents with switching topology and time-delays," IEEE Trans. on Automat. Contr., vol. 49, pp. 1520-1533, 2004.

[14] A. Sarlette, "Geometry and symmetries in coordination control," Ph.D. dissertation, University of Liège, Belgium, Liège, Belgium, 6 January 2009.

[15] N. Chopra and M. W. Spong, "On exponential synchronization of Kuramoto oscillators," IEEE Trans. on Automat. Contr., vol. 54, no. 2, pp. 353-357, 2009.

[16] J. A. Acebrón, L. L. Bonilla, C. J. P. Vicente, F. Ritort, and R. Spigler, "The Kuramoto model: A simple paradigm for synchronization phenomena," Reviews of modern physics, vol. 77, pp. 137-185, 2005.

[17] D. Aeyels and J. Rogge, "Existence of partial entrainment and stability of phase locking behavior of coupled oscillator," Progress of Theoretical Physics, vol. 112, no. 6, pp. 921-942, 2004.

[18] A. Jadbabaie, N.Motee, and M. Barahona, "On the stability of the Kuramoto model of coupled nonlinear oscillators," Proc. American Control Conference, vol. 5, pp. 4296-4301, 2004.

[19] J. van Hemmen and W. Wreszinski, "Lyapunov function for the Kuramoto model on nonlinearly coupled oscillators," Journal of Statistical Physics, vol. 72, pp. 145-166, 1993.

[20] E. Brown, P. Holmes, and J. Moehlis, "Globally coupled oscillator networks," in Perspectives and Problems in Nonlinear Science: A Celebratory Volume in Honor of Larry Sirovich, 2003.

[21] C. G. Assisi, V. K. Jirsa, and J. A. S. Kelso, "Synchrony and clustering in heterogeneous networks with global coupling and parameter dispersion," Phys. Rev. Lett., vol. 94, no. 1, 2005.

[22] A. L. Fradkov, Cybernietical Physics. From Control of Chaos to Quantum Control, J. S. Kelso, Ed. Berlin Heidelberg: SpringerVerlag, 2007.

[23] A. Pikovsky, M. Rosenblum, and J. Kurths, Synchronization: A Universal Concept in Nonlinear Sciences. Cambridge, United Kingdom: Cambridge Nonlinear Science Series, 2001.

[24] N. Chopra and M. W. Spong, "On synchronization of Kuramoto oscillators," in Proc. 44th. IEEE Conf. Decision Contr., 2005.

[25] B. C. Daniels, "Synchronization of globally coupled nonlinear oscillators: the rich behavior of the Kuramoto model," Available at http://go. owu.edu/p̃hysics/StudentResearch/2005/BryanDaniels/kuramoto_paper.pdf, 2005.

[26] Y. Maistrenko, O. Popovych, and P. Tass, "Desynchronization and chaos in the Kuramoto model," Lect. Notes Phys., vol. 671, pp. 285306, 2005.

[27] K. Pyragas, O. V. Popovich, and P. A. Tass, "Controlling synchrony in oscillatory networks with a separate stimulation-registration setup," EPL, vol. 80, no. 4, 2008. 
[28] N. Tukhlina, M. Rosenblum, A. Pikovsky, and J. Kurths, "Feedback suppression of neural synchrony by vanishing stimulation," Physical Review E, vol. 75, no. 1, p. 011918(8), 2007.

[29] P. Tass, "A model of desynchronizing deep brain stimulation with a demand-controlled coordinated reset of neural subpopulations," Biol. Cybern., vol. 89, pp. 81-88, 2003.

[30] C. Hauptmann, O. Popovych, and P. Tass, "Delayed feedback control of synchronization in locally coupled neuronal networks," Neurocomputing, vol. 65, pp. 759-767, 2005.

[31] A.Franci, A. Chaillet, and W. Pasillas-Lépine, "Existence of phaselocking in coupled kuramoto oscillators under real mean-field feedback with applications to Deep Brain Stimulation," Submitted to the Proc. 49th. IEEE Conf. Decision Contr., 2010.

[32] C. U. D. Cumin, "Generalising the Kuramoto model for the study of neuronal synchronisation in the brain," Physica D, vol. 226, pp. 181-196, 2007.

[33] R. Sepulchre, D. Paley, and N. E. Leonard, "Stabilization of planar collective motion with limited communication," IEEE Transactions on Automatic Control, vol. 53, no. 3, pp. 706-719, 2008.

[34] A. Loria and E. Panteley, "Cascade nonlinear time-varying systems: analysis and design," in Advanced topics in control systems theory, ser. Lecture Notes in Control and Information Sciences, F. LamnabhiLagarrigue, A. Loria, and E. Panteley, Eds. Springer Verlag, 2005.

[35] E. Sontag and Y. Wang, "New characterizations of input-to-state stability," IEEE Trans. on Automat. Contr., vol. 20, 1999.

[36] E. Sontag, "Remarks on stabilization and Input-to-State stability," in Proc. 28th. IEEE Conf. Decision Contr., 1989, pp. 1376-1378.

[37] I. J. Malkin, "Theory of stability of motion," U.S. Atomic energy commission, Tech. Rep., 1958.

[38] M. Rodriguez-Oroz, J. Obeso, A. Lang, J.-L. Houeto, P. Pollak, S. Rehncrona, J. Kulisevsky, A. Albanese, J. Volkmann, M. Hariz N. Quinn, J. Speelman, J. Guridi, I. Zamarbide, A. Gironell, J. Molet, B. Pascual-Sedano, B. Pidoux, A. Bonnet, Y. Agid, J. Xie, A. Benabid, A. Lozano, J. Saint-Cyr, L. Romito, M. Contarino, M. Scerrati, V. Fraix, and N. V. Blercom, "Bilateral deep brain stimulation in Parkinson's disease: a multicentre study with 4 years follow-up,' Brain, vol. 128, pp. 2240-2249, 2005.

[39] A. Chaillet and A. Loría, "Uniform global practical asymptotic stability for non-autonomous cascaded systems," European Journal of Control, vol. 12, no. 6, pp. 595-605, 2006.

[40] H. Khalil, Nonlinear systems. New York: Prentice Hall, 3rd ed., 2001.

[41] A.Isidori, Nonlinear control system II. Springer Verlag, 1999.

[42] E. Sontag and Y. Wang, "On characterizations of the input-to-state stability property," Syst. \& Contr. Letters, vol. 24, pp. 351-359, 1995.

[43] G. Bliss, Lectures on the calculus of variations. Chicago Univ. Press, 1947. 